\title{
Optics modification of the electron collector for the Relativistic Heavy Ion Collider Electron Beam Ion Source
}

\author{
A. Pikin, J.G. Alessi, E.N. Beebe, \\ D. Raparia, L. Snydstrup
}

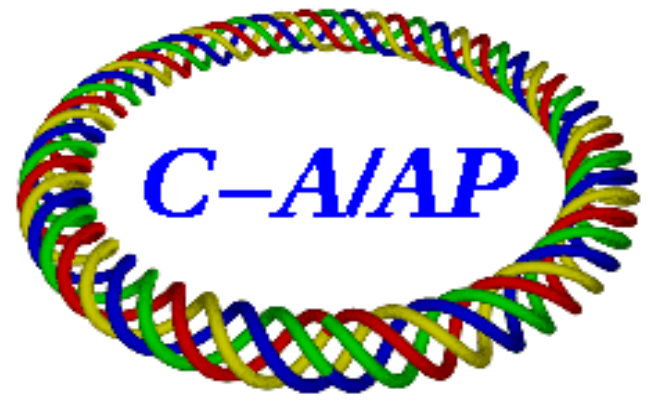

Collider-Accelerator Department Brookhaven National Laboratory Upton, NY 11973

Notice: This document has been authorized by employees of Brookhaven Science Associates, LLC under Contract No. DE-AC02-98CH10886 with the U.S. Department of Energy. The United States Government retains a nonexclusive, paid-up, irrevocable, world-wide license to publish or reproduce the published form of this document, or allow others to do so, for United States Government purposes. 


\title{
Optics modification of the electron collector for the Relativistic Heavy Ion Collider Electron Beam Ion Source
}

\author{
A. Pikin, J. G. Alessi, E. N. Beebe, D. Raparia, L. Snydstrup \\ Collider-Accelerating Department, \\ Brookhaven National Laboratory
}

\begin{abstract}
The Electron Beam Ion Source (EBIS) for the Relativistic Heavy Ion Collider (RHIC) at the Brookhaven National Laboratory (BNL) operates with electron beam current up to $10 \mathrm{~A}$ and frequency up to 4-5 Hz. The observation of the vacuum conditions and the temperature of the electron repeller electrode inside the electron collector (EC) points to heavy heating of this electrode by the backscattered electrons generated by the primary electron beam on the inner surfaces of the EC. The ion optics adjacent to the EC operates at potentials of up to $50 \mathrm{kV}$ and higher and the flashes of the desorbed gas may cause discharges and have detrimental effect on the vacuum conditions in the ion trap, and possible instability of the extracted ion beam. The proposed modification of the EC optics has a goal of eliminating the heating of the internal EC electrodes with preservation of the existing structure of the ion optics. The results of computer simulations of the electron beam dissipation and ion extraction are presented.
\end{abstract}

\section{Introduction}

RHIC EBIS provides a variety of ions from He to U with charge states acceptable for the preinjector and Booster (charge to mass ratio $\geq 1 / 6$ ). With the existing ion injection system, consisting of two external primary Hollow Cathode ion sources with appropriate electrostatic optics, EBIS can provide several ion species for different users on a pulse-to-pulse basis. The intensity of the ion beam in the absence of the residual gas contamination (which is practically a case for RHIC EBIS) is determined by the capacity of the ion trap, which is $5.1^{*} 10^{12}$ elementary charges for the electron current $10 \mathrm{~A}$ and electron energy $20 \mathrm{keV}$ (50\% of the total electron charge in the trap), and by the abundance of the ions with the required mass and charge state in the spectrum of the extracted ion beam. The duration of the ion pulse on the half of amplitude is $(5-10) \mu$ s depending on the Booster injection requirement. A comprehensive optical, thermal and stress analysis of the RHIC EBIS electron collector can be found in [1], optical simulations and design of RHIC EBIS main components have been published in [2-5]. With high average power of the electron beam, which can be reached either with $1 \mathrm{~Hz}$ operation and $200 \mathrm{~ms}$ electron beam pulse length or $4 \mathrm{~Hz}$ with 50 $\mathrm{ms}$, the temperature of the electron repeller electrode reaches up to $450 \mathrm{C}$, measured with thermocouple attached directly to this electrode in its middle. Heating of electrodes during the electron pulse results in a pressure increase inside the electron collector chamber up to 3 times in a range $\left(1^{*} 10^{-8}-2^{*} 10^{-9}\right)$ Tor. Both such pressure level and pressure variations are detrimental for EBIS performance. Improving the vacuum conditions in the EC region of RHIC EBIS is a goal of the proposed modification. 


\section{Existing EC geometry}

The schematic of the original existing EC optical structure is presented in Fig. 1.

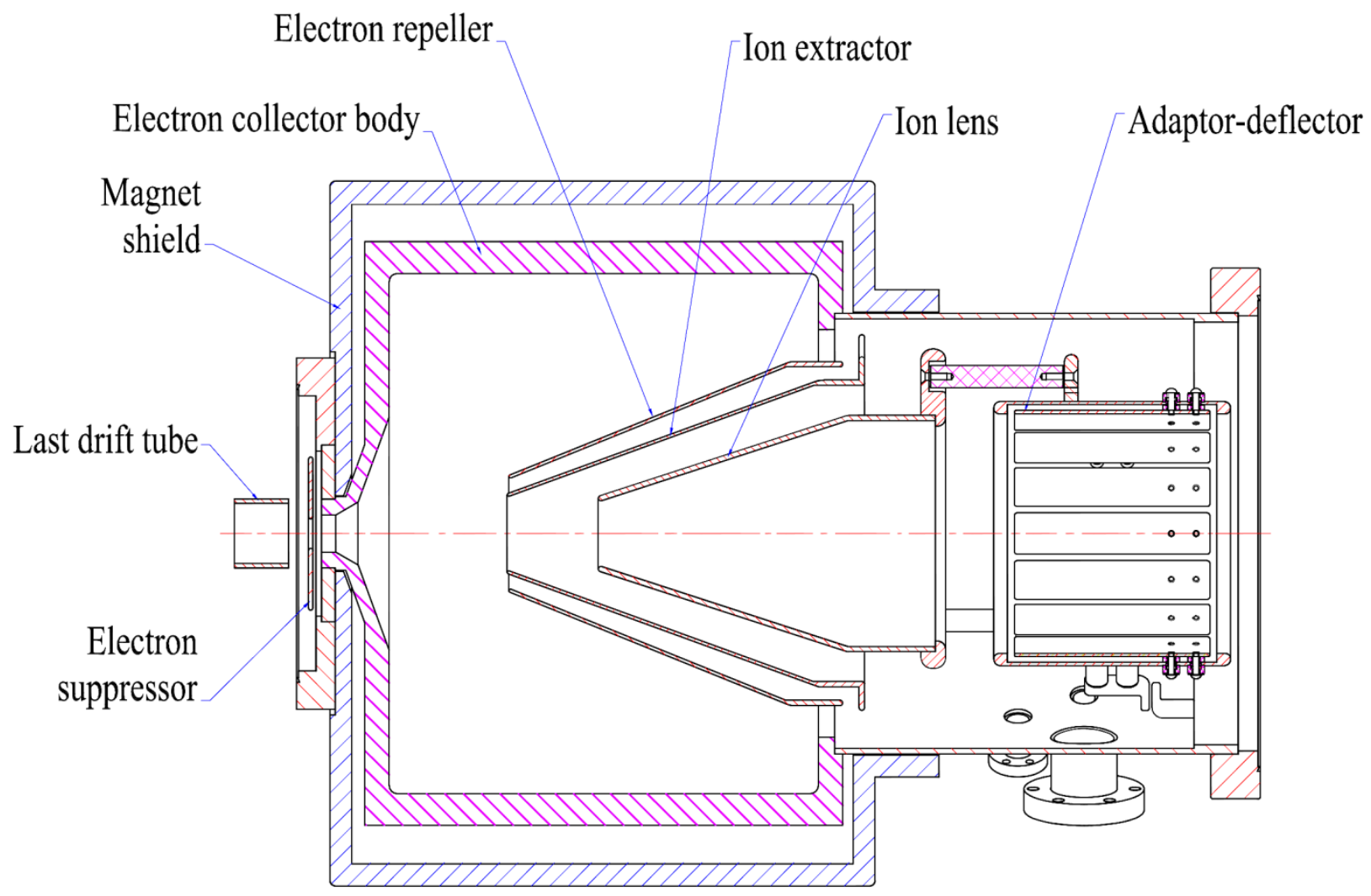

Fig. 1. Optical geometry of the original RHIC EBIS electron collector

To satisfy the requirement to dissipate power of the electron beam up to $300 \mathrm{~kW}$ within a limited surface of the electron collector the density of the electron current deposition on its surface should be distributed with maximum possible uniformity. The spikes in power density distribution could cause either thermal damage or substantial reduction of its life time due to elevated fatigue.

A choice of such geometry with ion extractor edge located close to the EC entrance aperture and with a shielding electrode over the ion extractor was determined by the two main considerations:

- To start decoupling of the ion beam from the electron beam on the early stage of the electron beam expansion, so that the ion beam has a small diameter at the entrance into ion optics and therefore will be less prone to the spherical aberration with the available size of the ion optical elements.

- To stretch the electron beam over the longer portion of the water-cooled cylindrical part of the EC by shielding the repulsing action of the ion extractor by the additional shielding electrode (electron repeller), so that the electron beam is distributed over larger watercooled area.

The backscattered electrons were not taken into account in this design and caused substantial heating of the electron repeller electrode. To reduce this heating a bias voltage of $-(2.5-3.0) \mathrm{kV}$ has to be applied to the repeller electrode. The reduction of the repeller load with biasing the repeller 
electrode came at a price of pushing the primary electron beam backwards, which caused an increased power density on the EC cylindrical surface compare to an initial design distribution with not biased repeller.

A new run of TRAK simulations of the electron distribution on EC surfaces has been done with a model of backscattered electrons, which have a cosine angular distribution with respect to the normal to the emitting surface and the energy spectrum of the emitted backscattered electrons from [9]. Since the dominating component of our $\mathrm{Cu}-\mathrm{Zr}$ alloy is $\mathrm{Cu}$ the coefficient of backscattering in these simulations was taken 0.3 over the energy range of $(0.05-11) \mathrm{keV}$. Only one generation of the backscattered electrons has been used since the effect of them on power density distributions was found to be relatively small.

The simulation of the primary and secondary electrons transmission for the original EC is presented in Fig. 2.

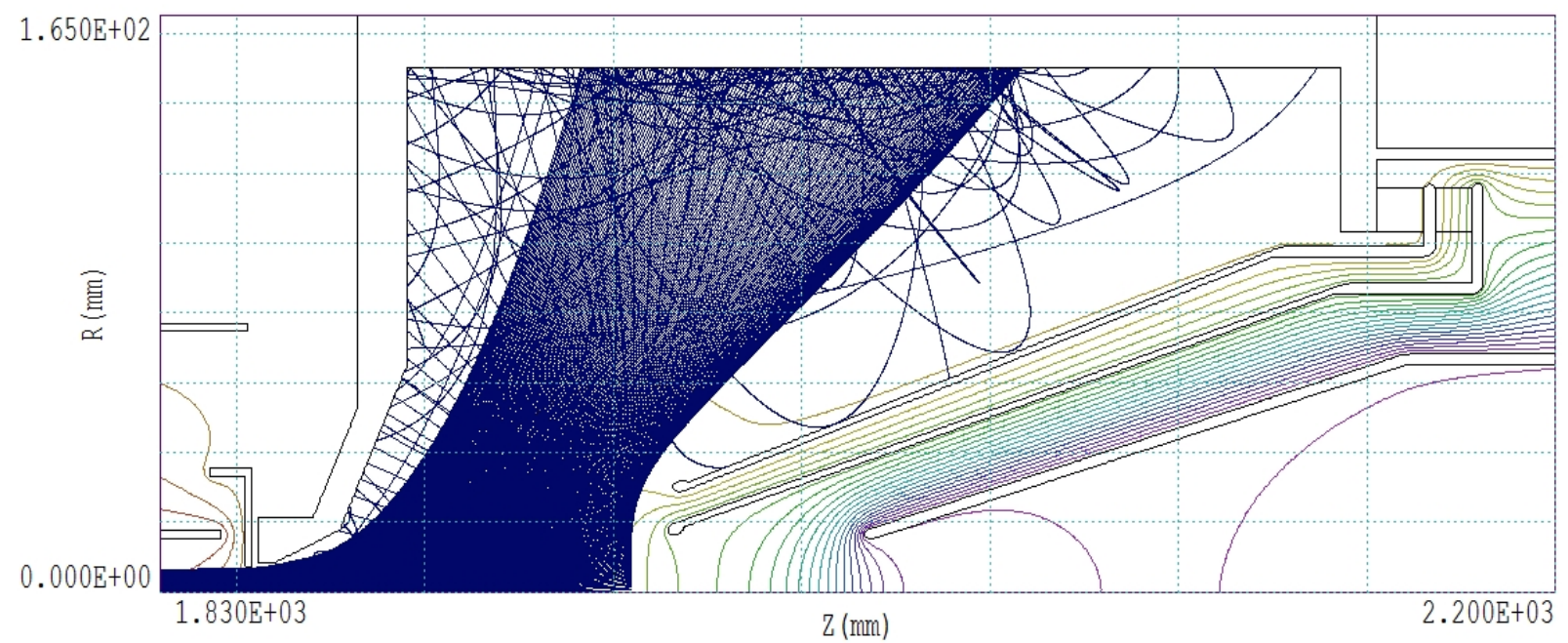

Fig. 2. Trajectory simulations of the primary and backscattered electrons in the original version of the RHIC EBIS EC. $\mathrm{I}_{\mathrm{el}}=10.0 \mathrm{~A}, \mathrm{E}_{\mathrm{el}}=11.0 \mathrm{keV}, \mathrm{U}_{\text {repel }}=-2.6 \mathrm{kV}$.

The axial power distributions of the primary and backscattered components of the electron beam are presented in Fig.3.

From both figures one can see that the backscattered electrons are largely confined to the areas of their generation with the exception of vertical wall, which still have a relatively low power density for our EC design.

The radial distributions of the power density deposited by the same components of the electron beam on the vertical wall of the EC are presented in Fig. 4. The modification of the power distribution on the vertical wall by the backscattered electrons is well visible. The power density levels on the vertical wall for both primary and backscattered components of the electron beam are much smaller than for the cylindrical wall and do not present any problem for removing the heat from EC.

The simulated values of the electron current returned from the EC backwards to the plane of origin for models including and excluding the backscattered electrons are the same: $0.4 \mathrm{~mA}$ and presents a 
reflected near-axis part of the primary electron beam. No reflected flux of the backscattered electrons was noticed in this simulation with the total number of the backscattered electron trajectories of 2000 .

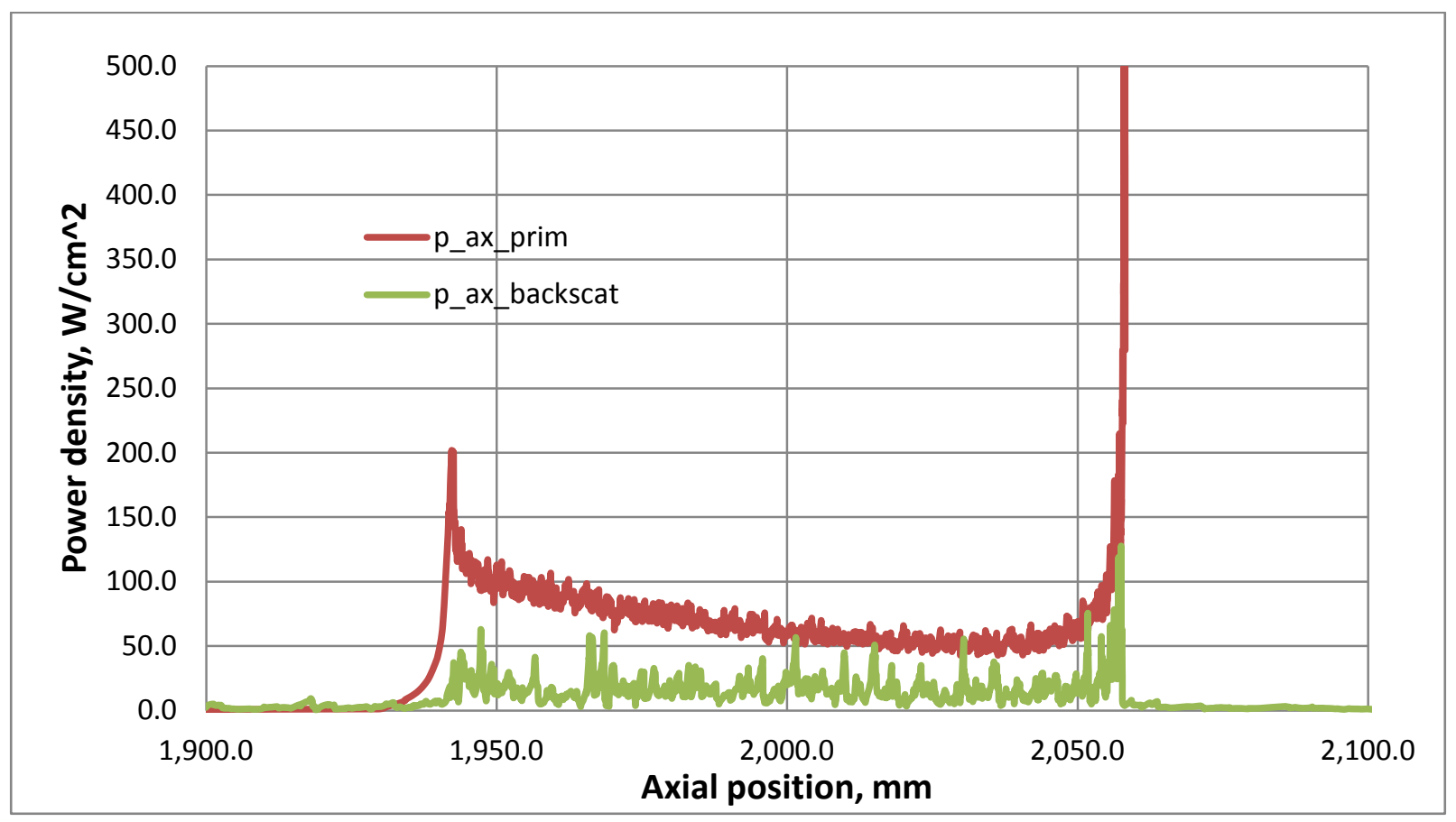

Fig. 3. Axial power density distributions of the primary and backscattered electrons in the existing EC. Primary beam parameters: $\mathrm{I}_{\mathrm{el}}=10.0 \mathrm{~A}, \mathrm{E}_{\mathrm{el}}=11.0 \mathrm{keV}, \mathrm{U}_{\text {repel }}=-2.6 \mathrm{kV}$.

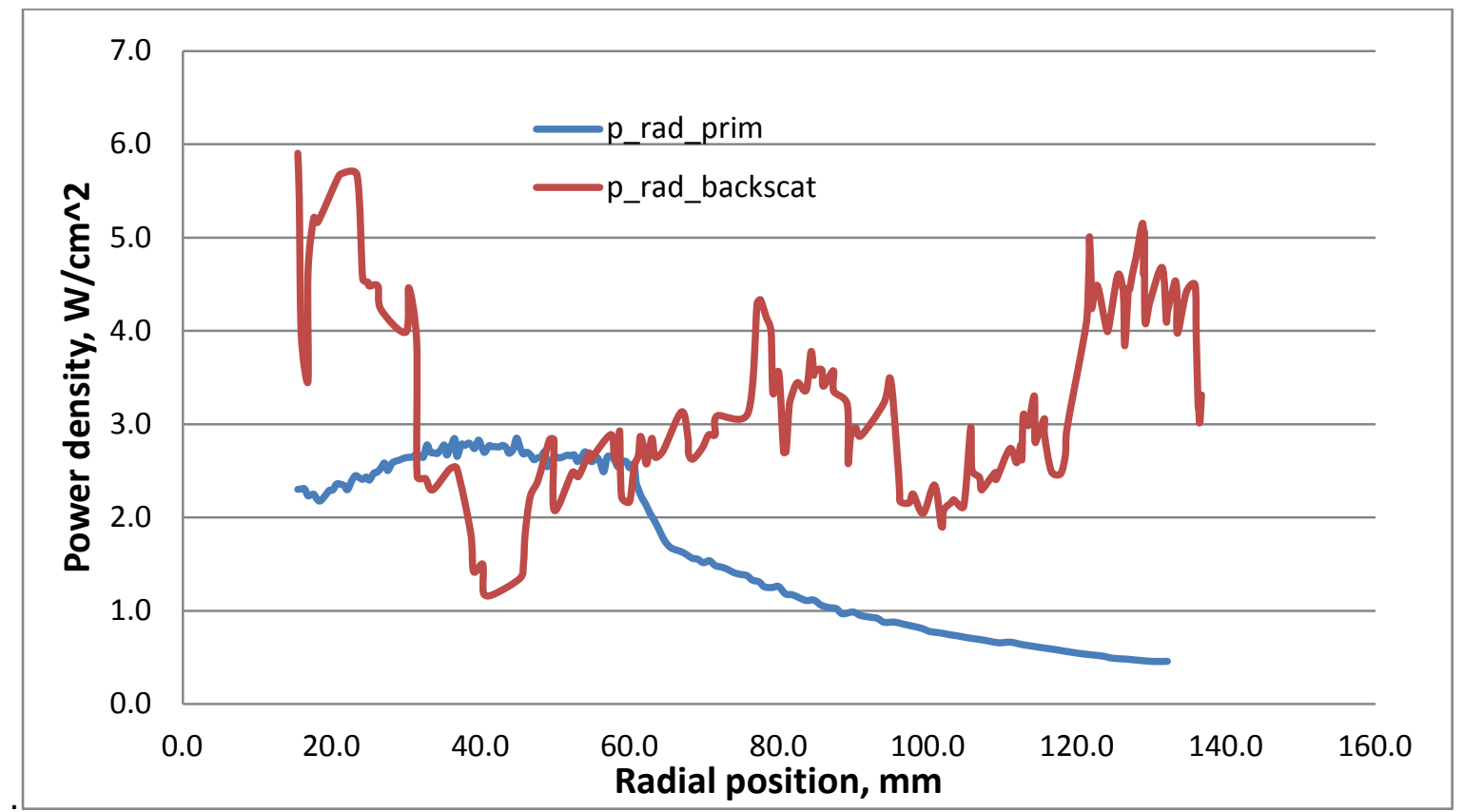

Fig. 4. Radial distributions of power density for primary and backscattered components of the electron beam for primary beam parameters: $\mathrm{I}_{\mathrm{el}}=10.0 \mathrm{~A}, \mathrm{E}_{\mathrm{el}}=11.0 \mathrm{keV}, \mathrm{U}_{\text {repel }}=-2.6 \mathrm{kV}$. 
For $\mathrm{I}_{\mathrm{el}}=10.0 \mathrm{~A}, \mathrm{E}_{\mathrm{el}}=11.0 \mathrm{keV}, \mathrm{U}_{\text {repel }}=-2.6 \mathrm{kV}$ the simulated value of the peak power of the backscattered electrons hitting the biased repeller electrode is 482.1 watt. The temperature of this electrode is measured with a thermocouple attached to its surface and shielded from the direct hit by the electrons. With electron current $9.0 \mathrm{~A}$, beam pulse length $58 \mathrm{~ms}$, frequency $5 \mathrm{~Hz}$ the observed temperature of the repeller electrode was $450^{\circ} \mathrm{C}$. Such equilibrium temperature with radiative heat exchange can be obtained with the value of the equivalent emissivity of the emitting surface 0.07 . The material of the repeller is stainless steel and the internal part of it is thermally shielded with a "floating" polished stainless steel ion lens (see Fig. 1). With increased negative bias on the repeller electrode the temperature of this electrode decreases, but one should expect the power density on the EC cylindrical surface to go up.

The pressure of the residual gas in the $\mathrm{EC}$ vacuum chamber during the electron pulse typically increases 3 times in a range $\left(2^{*} 10^{-9}-1^{*} 10^{-8}\right)$ Tor due to pulsed bombardment of the internal EC surfaces by all electron components. The hot repeller electrode contributes to this pressure. Considering the high electric field environment of the extracting ion optics and the vicinity of the ion trap, which is sensitive to the residual gas, the heating of the repeller can be considered detrimental for both high voltage holdoff and the stability of the EBIS operation. Elimination of this electrode is a goal of this modification.

\section{Modification of the electron collector optics}

Just the elimination of the electron repeller electrode with all other electrodes intact would extend the electric field, which reflects electrons into the volume of the electron collector, pushing the primary electrons close to the entrance diaphragm and onto the vertical wall with limited cooling capability. On the other hand, if the ion extractor is moved further away from the EC entrance diaphragm, its reflecting field in the region of the electron beam divergence can become acceptable and the elimination of the electron repeller can be done with the existing electron collector. A proposed modification of the EC electrode system is presented in Fig. 5.

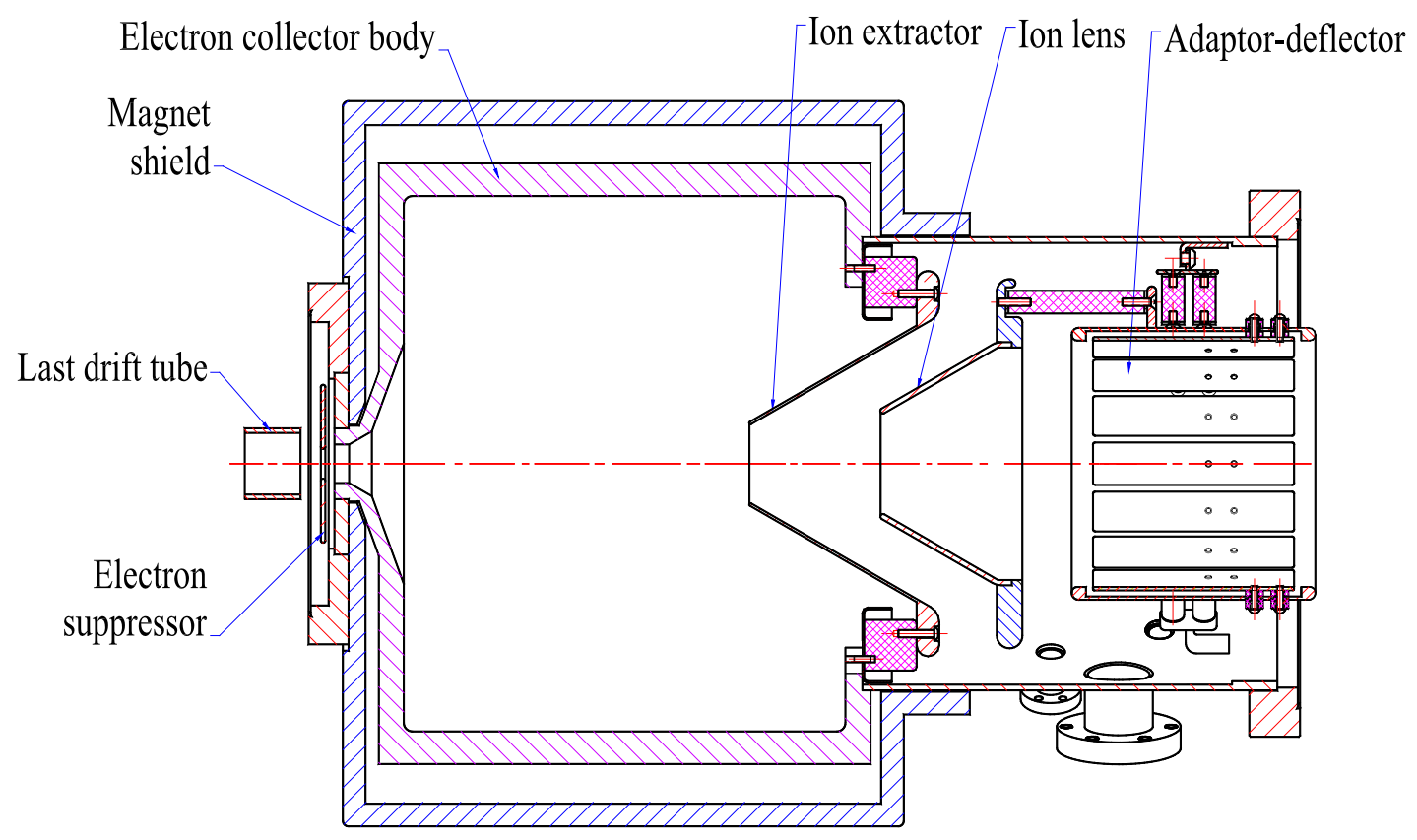

Fig. 5. Electrode geometry of the modified RHIC EBIS electron collector. 
Considering that the properties of the existing ion extraction/ion injection EC optics are acceptable, the general structure of the electrodes remains the same with some modification in dimensions and positions. It was possible to preserve the existing 16-pole adaptor/deflector.

The new ion extractor has approximately the same diameter of the entrance aperture (44 $\mathrm{mm})$ and the aperture of the new ion lens is larger taking into account an increased initial ion beam diameter compare to the existing optics. The HV holdoff of electrodes in an upgraded version is expected to be better because of an improved supports and reduced critical electric fields.

The simulated trajectories of the primary and backscattered electrons in a modified EC are presented in Fig. 6.

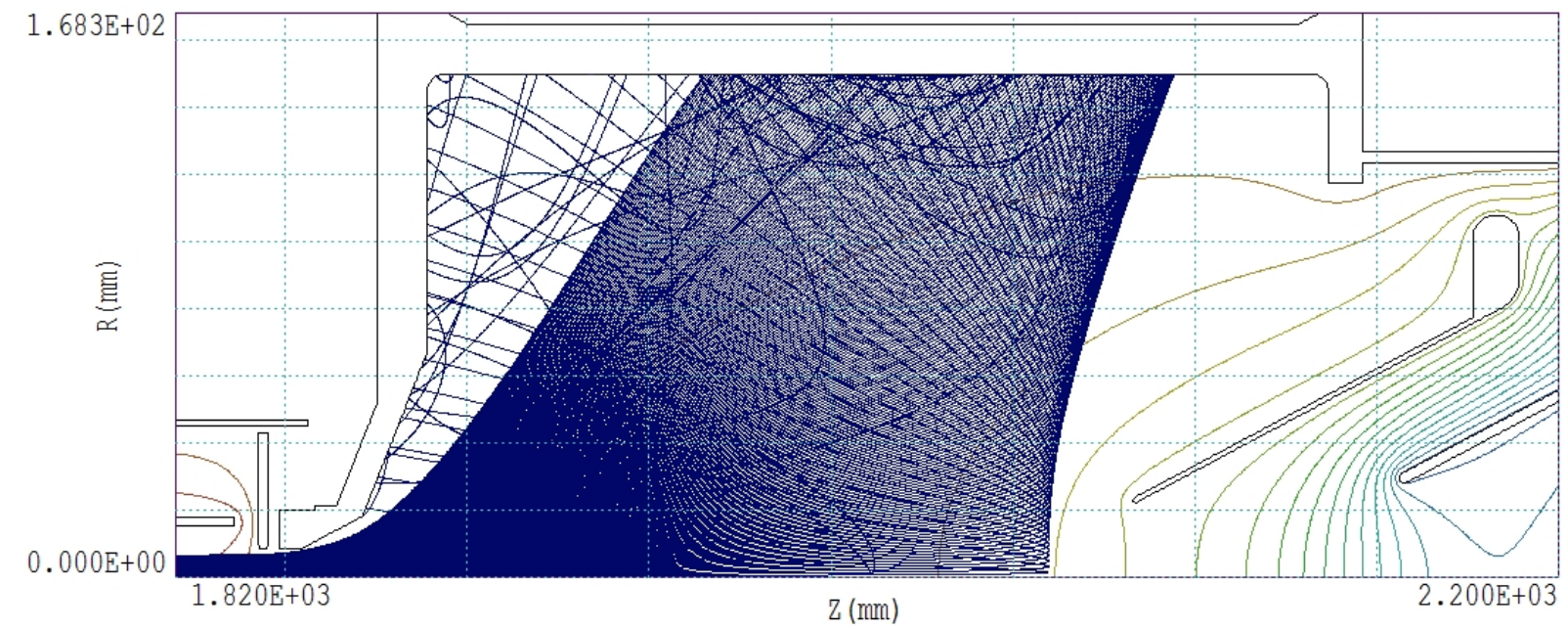

Fig. 6. Simulated trajectories of the primary electrons and of the first generation of backscattered electrons in RHIC EBIS EC with new optical geometry. $\mathrm{I}_{\mathrm{el}}=10.0 \mathrm{~A}, \mathrm{E}_{\mathrm{el}}=11.0 \mathrm{keV}$.

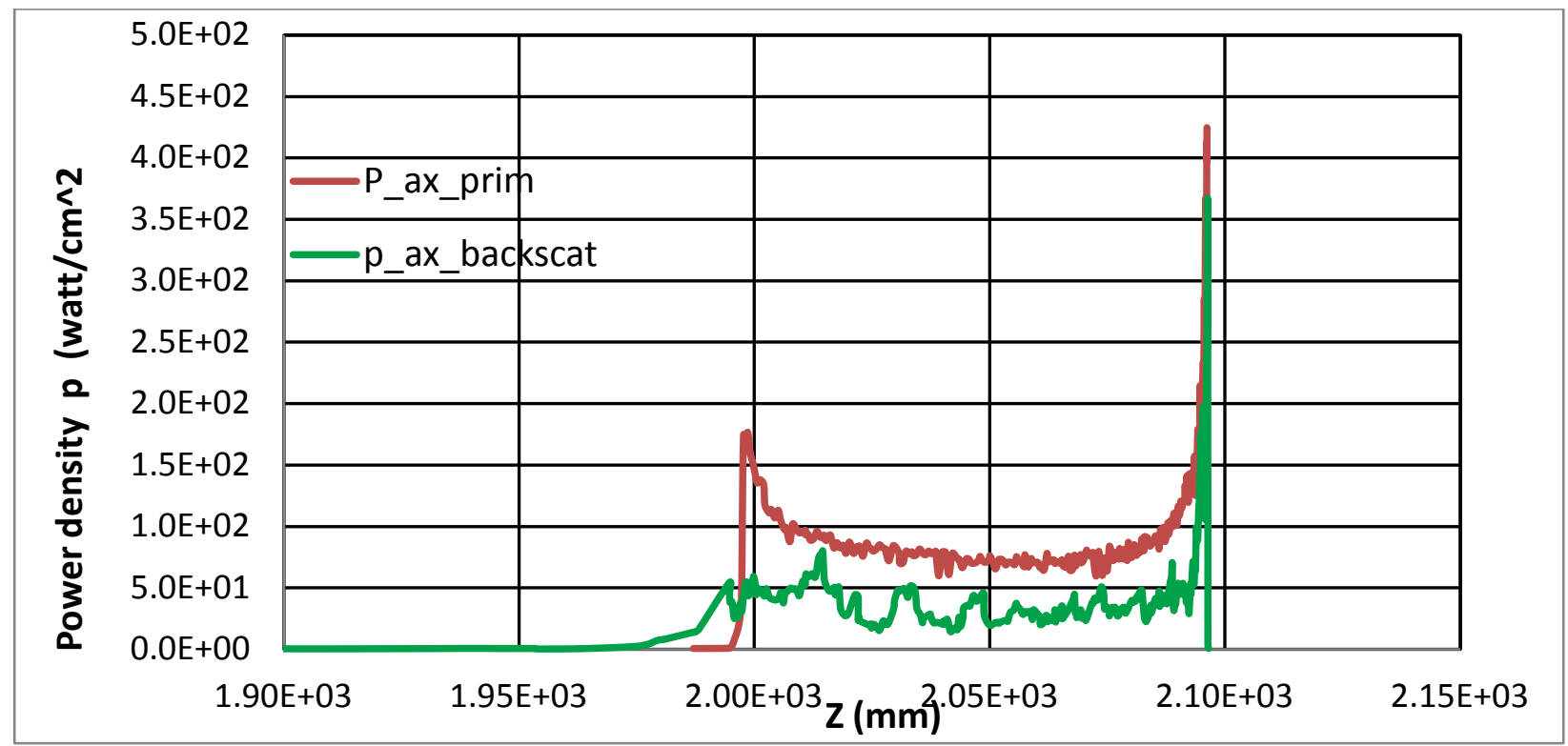

Fig. 7. Axial power density distributions of the primary and backscattered electrons in a new geometry of EC optics. Primary beam parameters: $\mathrm{I}_{\mathrm{el}}=10.0 \mathrm{~A}, \mathrm{E}_{\mathrm{el}}=11.0 \mathrm{keV}$. 
Similar to a previous case, the deposition of the backscattered electrons is mostly limited to the areas, which are bombarded by the primary electrons. The power density distribution on the cylindrical surface for a new geometry is presented in Fig. 7. For electron current $10 \mathrm{~A}$ the power density distribution on the cylindrical surface of EC remains approximately the same for both versions of EC optics, even that it shifts axially forward in a new version compare to the existing one. No effect on the reflecting of the primary electron backwards, resulting from this modification has been observed.

\section{Conclusion}

The proposed modification of the RHIC EBIS electron collector with purpose to eliminate the hot electron repeller electrode can be done with the existing electron collector body and its vacuum chamber. The simulated power dissipation on the electron collector walls remains similar to the original EC and the simulated flux of the reflected electrons did not increase. The 16-pole adaptor deflector remains intact and only its position has been changed. The HV design is more robust than the original one. Since the structure of the ion optical element did not change, only the dimensions did, the effect of optical elements in a modified version of EC on the injected and extracted ion beams remains similar to the original one according to our initial plans.

\section{References}

1. Alexander Pikin, Ahovi Kponou, Louis Snydstrup, Optical, Thermal and Stress Simulations of a 300-kwatt Electron Collector, BNL Tech Note C-A/AP/\#246, 2006

2. Alexander Pikin, James G. Alessi, Edward N. Beebe, Ahovi Kponou, Krsto Prelec, John Ritter, Louis Snydstrup, Analysis of a possible 20 A electron gun and collector design for the RHIC EBIS, Journal of Physics: Conference series 2, pp. 28-34, 2004

3. J. G. Alessi, E. Beebe, D. Graham, A. Kponou, K. Prelec, J. Ritter, V. Zajic, Design of an EBIS for RHIC, PAC-2003 Proceedings, V. 1, pp. 89-91, 2003

4. A. Pikin, J. G. Alessi, E. N. Beebe, A. Kponou, R. Lambiase, R. Lockey, D. Raparia, J. Ritter, L. Snydstrup and Y. Tan, RHIC EBIS: basics of design and status of commissioning, Proceedings of the 11-th International Symposium on Electron Beam ion Sources and Traps, Stockholm, 2010, in JINST, 5, C09003, 2010

5. A. Pikin, j. Alessi, E. Beebe, A. Kponou, M. Okamura, Ion optics of RHIC Electron Beam Ion Source, Rev. Sci. Instrum, 83, 02A50, 2012 


\title{
Optics modification of the electron collector for the Relativistic Heavy Ion Collider Electron Beam Ion Source
}

\author{
A. Pikin, J. G. Alessi, E. N. Beebe, D. Raparia, L. Snydstrup \\ Collider-Accelerating Department, \\ Brookhaven National Laboratory
}

\begin{abstract}
The Electron Beam Ion Source (EBIS) for the Relativistic Heavy Ion Collider (RHIC) at the Brookhaven National Laboratory (BNL) operates with electron beam current up to $10 \mathrm{~A}$ and frequency up to 4-5 Hz. The observation of the vacuum conditions and the temperature of the electron repeller electrode inside the electron collector (EC) points to heavy heating of this electrode by the backscattered electrons generated by the primary electron beam on the inner surfaces of the EC. The ion optics adjacent to the EC operates at potentials of up to $50 \mathrm{kV}$ and higher and the flashes of the desorbed gas may cause discharges and have detrimental effect on the vacuum conditions in the ion trap, and possible instability of the extracted ion beam. The proposed modification of the EC optics has a goal of eliminating the heating of the internal EC electrodes with preservation of the existing structure of the ion optics. The results of computer simulations of the electron beam dissipation and ion extraction are presented.
\end{abstract}

\section{Introduction}

RHIC EBIS provides a variety of ions from He to U with charge states acceptable for the preinjector and Booster (charge to mass ratio $\geq 1 / 6$ ). With the existing ion injection system, consisting of two external primary Hollow Cathode ion sources with appropriate electrostatic optics, EBIS can provide several ion species for different users on a pulse-to-pulse basis. The intensity of the ion beam in the absence of the residual gas contamination (which is practically a case for RHIC EBIS) is determined by the capacity of the ion trap, which is $5.1^{*} 10^{12}$ elementary charges for the electron current $10 \mathrm{~A}$ and electron energy $20 \mathrm{keV}$ (50\% of the total electron charge in the trap), and by the abundance of the ions with the required mass and charge state in the spectrum of the extracted ion beam. The duration of the ion pulse on the half of amplitude is $(5-10) \mu$ s depending on the Booster injection requirement. A comprehensive optical, thermal and stress analysis of the RHIC EBIS electron collector can be found in [1], optical simulations and design of RHIC EBIS main components have been published in [2-5]. With high average power of the electron beam, which can be reached either with $1 \mathrm{~Hz}$ operation and $200 \mathrm{~ms}$ electron beam pulse length or $4 \mathrm{~Hz}$ with 50 $\mathrm{ms}$, the temperature of the electron repeller electrode reaches up to $450 \mathrm{C}$, measured with thermocouple attached directly to this electrode in its middle. Heating of electrodes during the electron pulse results in a pressure increase inside the electron collector chamber up to 3 times in a range $\left(1^{*} 10^{-8}-2^{*} 10^{-9}\right)$ Tor. Both such pressure level and pressure variations are detrimental for EBIS performance. Improving the vacuum conditions in the EC region of RHIC EBIS is a goal of the proposed modification. 


\section{Existing EC geometry}

The schematic of the original existing EC optical structure is presented in Fig. 1.

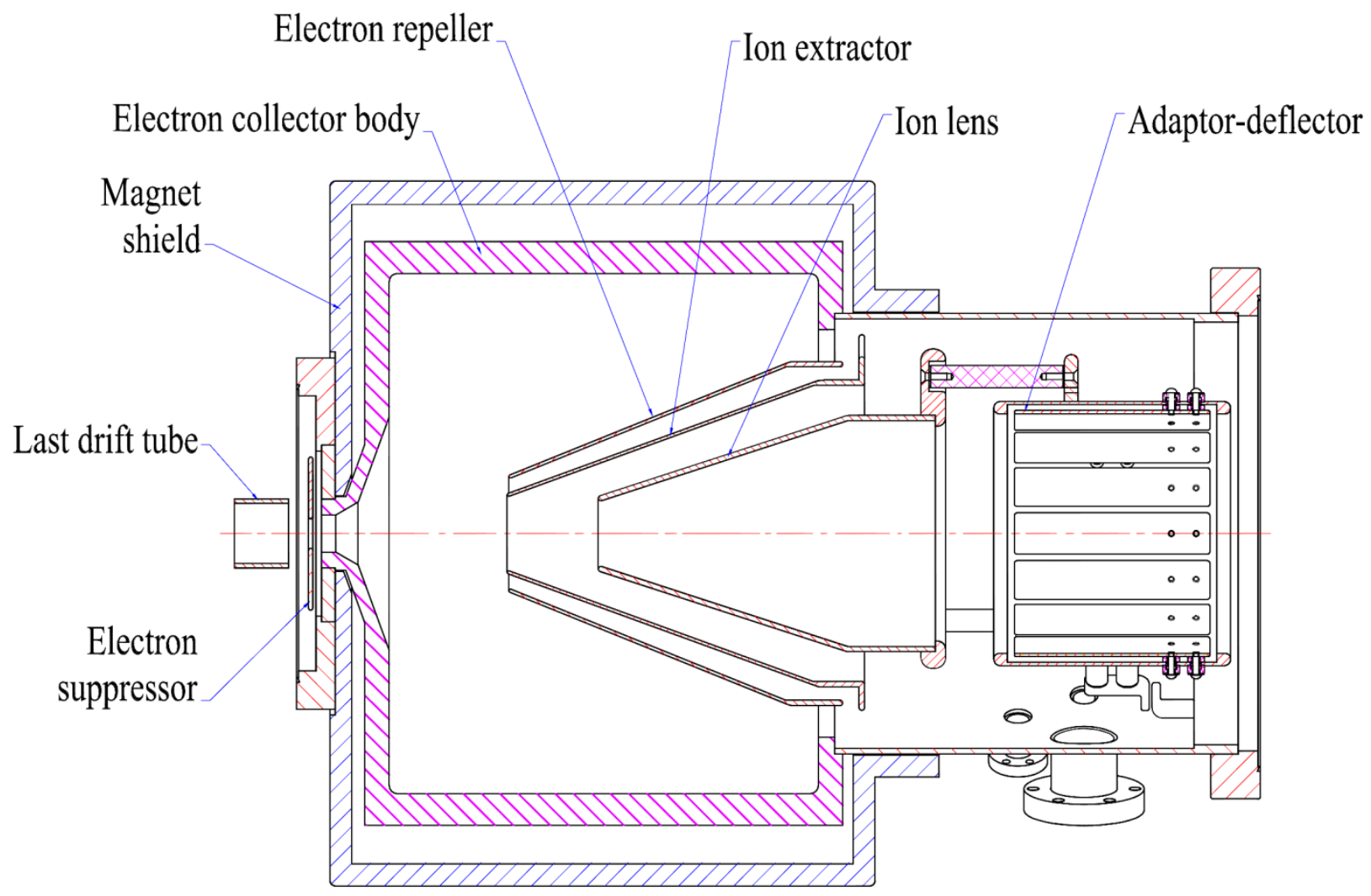

Fig. 1. Optical geometry of the original RHIC EBIS electron collector

To satisfy the requirement to dissipate power of the electron beam up to $300 \mathrm{~kW}$ within a limited surface of the electron collector the density of the electron current deposition on its surface should be distributed with maximum possible uniformity. The spikes in power density distribution could cause either thermal damage or substantial reduction of its life time due to elevated fatigue.

A choice of such geometry with ion extractor edge located close to the EC entrance aperture and with a shielding electrode over the ion extractor was determined by the two main considerations:

- To start decoupling of the ion beam from the electron beam on the early stage of the electron beam expansion, so that the ion beam has a small diameter at the entrance into ion optics and therefore will be less prone to the spherical aberration with the available size of the ion optical elements.

- To stretch the electron beam over the longer portion of the water-cooled cylindrical part of the EC by shielding the repulsing action of the ion extractor by the additional shielding electrode (electron repeller), so that the electron beam is distributed over larger watercooled area.

The backscattered electrons were not taken into account in this design and caused substantial heating of the electron repeller electrode. To reduce this heating a bias voltage of $-(2.5-3.0) \mathrm{kV}$ has to be applied to the repeller electrode. The reduction of the repeller load with biasing the repeller 
electrode came at a price of pushing the primary electron beam backwards, which caused an increased power density on the EC cylindrical surface compare to an initial design distribution with not biased repeller.

A new run of TRAK simulations of the electron distribution on EC surfaces has been done with a model of backscattered electrons, which have a cosine angular distribution with respect to the normal to the emitting surface and the energy spectrum of the emitted backscattered electrons from [9]. Since the dominating component of our $\mathrm{Cu}-\mathrm{Zr}$ alloy is $\mathrm{Cu}$ the coefficient of backscattering in these simulations was taken 0.3 over the energy range of $(0.05-11) \mathrm{keV}$. Only one generation of the backscattered electrons has been used since the effect of them on power density distributions was found to be relatively small.

The simulation of the primary and secondary electrons transmission for the original EC is presented in Fig. 2.

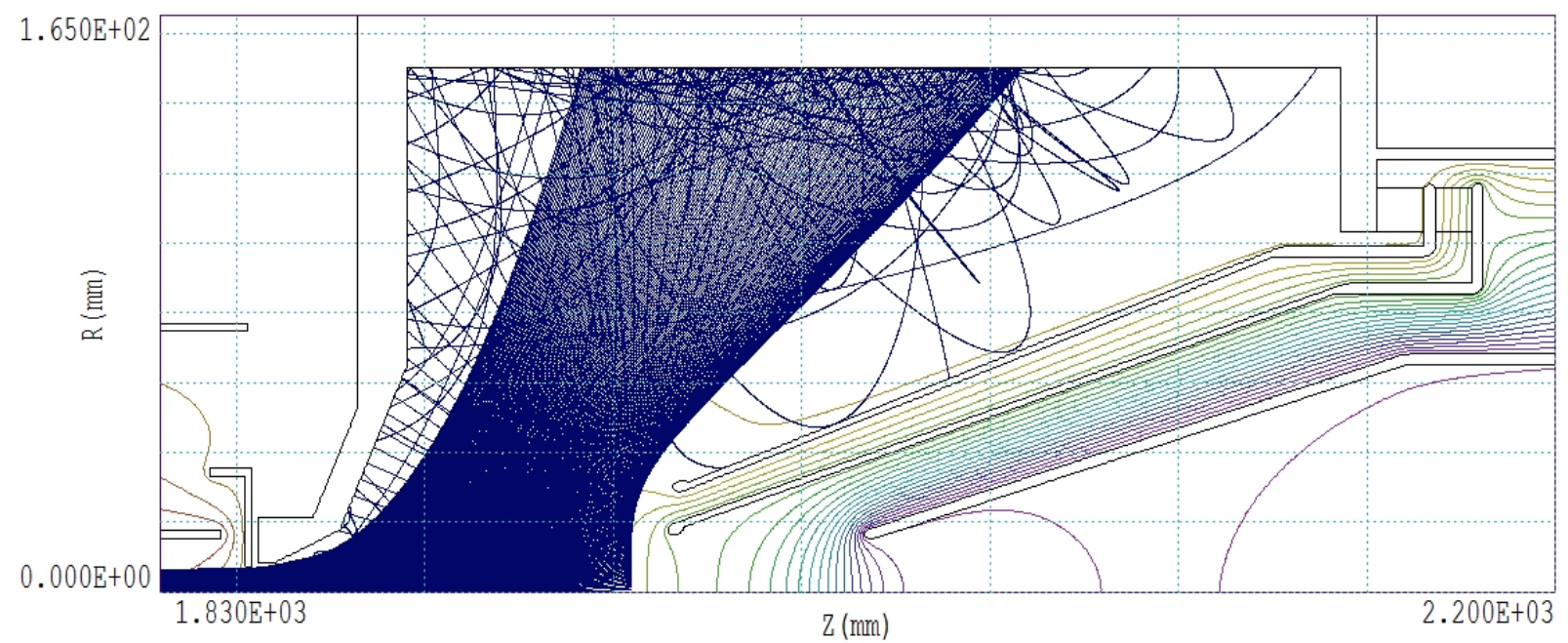

Fig. 2. Trajectory simulations of the primary and backscattered electrons in the original version of the RHIC EBIS EC. $\mathrm{I}_{\mathrm{el}}=10.0 \mathrm{~A}, \mathrm{E}_{\mathrm{el}}=11.0 \mathrm{keV}, \mathrm{U}_{\text {repel }}=-2.6 \mathrm{kV}$.

The axial power distributions of the primary and backscattered components of the electron beam are presented in Fig.3.

From both figures one can see that the backscattered electrons are largely confined to the areas of their generation with the exception of vertical wall, which still have a relatively low power density for our EC design.

The radial distributions of the power density deposited by the same components of the electron beam on the vertical wall of the EC are presented in Fig. 4. The modification of the power distribution on the vertical wall by the backscattered electrons is well visible. The power density levels on the vertical wall for both primary and backscattered components of the electron beam are much smaller than for the cylindrical wall and do not present any problem for removing the heat from EC.

The simulated values of the electron current returned from the EC backwards to the plane of origin for models including and excluding the backscattered electrons are the same: $0.4 \mathrm{~mA}$ and presents a 
reflected near-axis part of the primary electron beam. No reflected flux of the backscattered electrons was noticed in this simulation with the total number of the backscattered electron trajectories of 2000 .

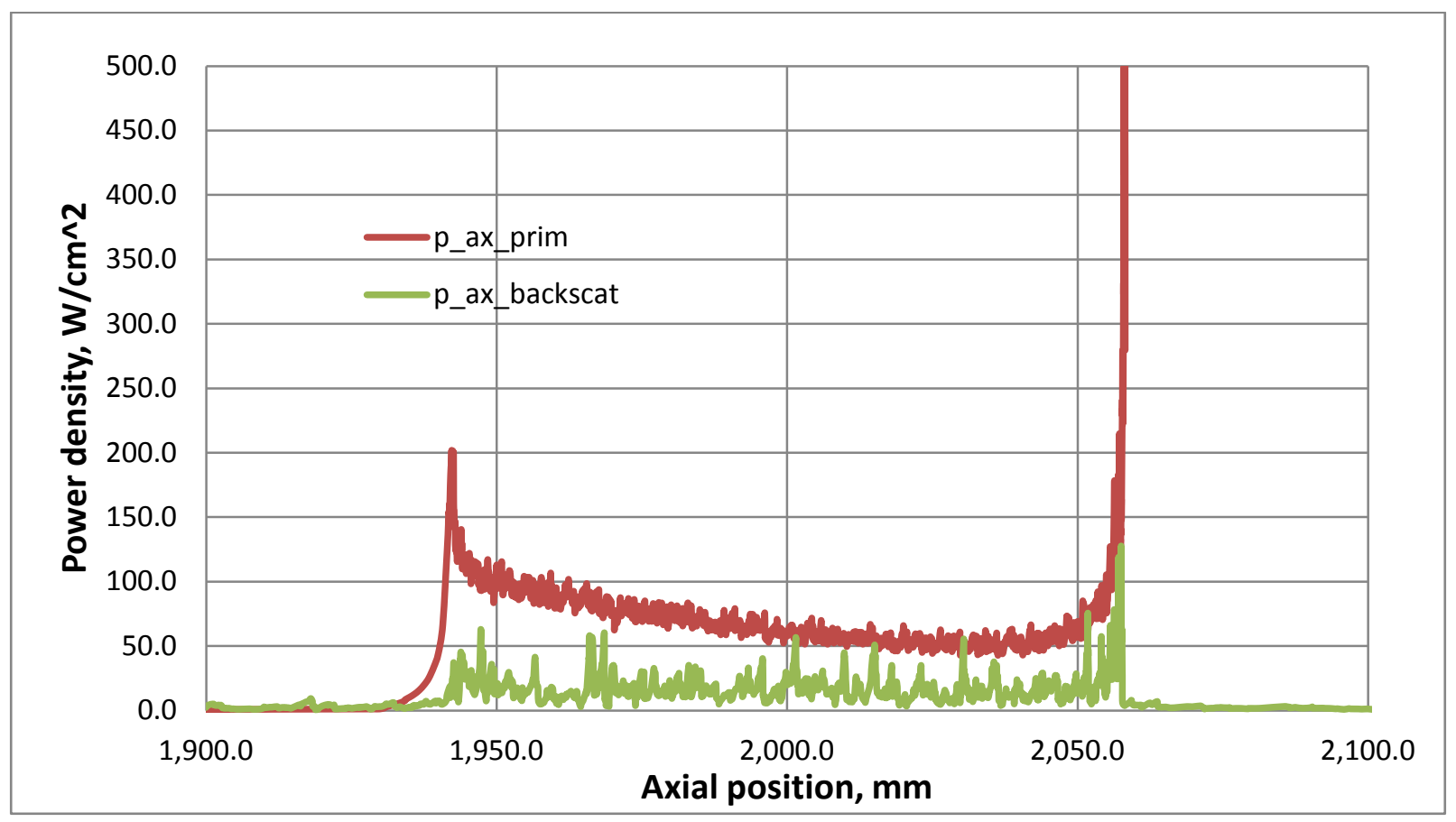

Fig. 3. Axial power density distributions of the primary and backscattered electrons in the existing EC. Primary beam parameters: $\mathrm{I}_{\mathrm{el}}=10.0 \mathrm{~A}, \mathrm{E}_{\mathrm{el}}=11.0 \mathrm{keV}, \mathrm{U}_{\text {repel }}=-2.6 \mathrm{kV}$.

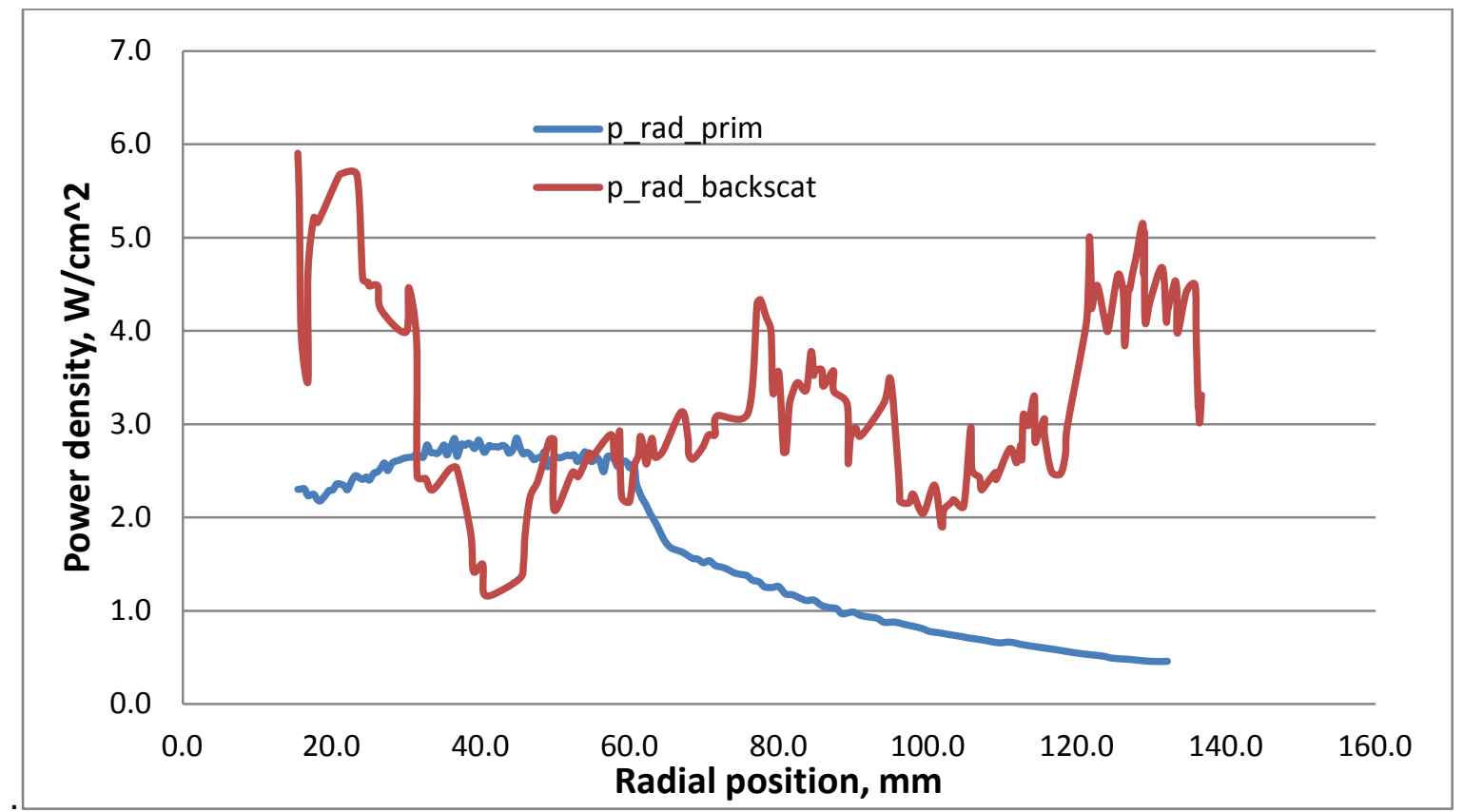

Fig. 4. Radial distributions of power density for primary and backscattered components of the electron beam for primary beam parameters: $\mathrm{I}_{\mathrm{el}}=10.0 \mathrm{~A}, \mathrm{E}_{\mathrm{el}}=11.0 \mathrm{keV}, \mathrm{U}_{\text {repel }}=-2.6 \mathrm{kV}$. 
For $\mathrm{I}_{\mathrm{el}}=10.0 \mathrm{~A}, \mathrm{E}_{\mathrm{el}}=11.0 \mathrm{keV}, \mathrm{U}_{\text {repel }}=-2.6 \mathrm{kV}$ the simulated value of the peak power of the backscattered electrons hitting the biased repeller electrode is 482.1 watt. The temperature of this electrode is measured with a thermocouple attached to its surface and shielded from the direct hit by the electrons. With electron current $9.0 \mathrm{~A}$, beam pulse length $58 \mathrm{~ms}$, frequency $5 \mathrm{~Hz}$ the observed temperature of the repeller electrode was $450^{\circ} \mathrm{C}$. Such equilibrium temperature with radiative heat exchange can be obtained with the value of the equivalent emissivity of the emitting surface 0.07 . The material of the repeller is stainless steel and the internal part of it is thermally shielded with a "floating" polished stainless steel ion lens (see Fig. 1). With increased negative bias on the repeller electrode the temperature of this electrode decreases, but one should expect the power density on the EC cylindrical surface to go up.

The pressure of the residual gas in the $\mathrm{EC}$ vacuum chamber during the electron pulse typically increases 3 times in a range $\left(2^{*} 10^{-9}-1^{*} 10^{-8}\right)$ Tor due to pulsed bombardment of the internal EC surfaces by all electron components. The hot repeller electrode contributes to this pressure. Considering the high electric field environment of the extracting ion optics and the vicinity of the ion trap, which is sensitive to the residual gas, the heating of the repeller can be considered detrimental for both high voltage holdoff and the stability of the EBIS operation. Elimination of this electrode is a goal of this modification.

\section{Modification of the electron collector optics}

Just the elimination of the electron repeller electrode with all other electrodes intact would extend the electric field, which reflects electrons into the volume of the electron collector, pushing the primary electrons close to the entrance diaphragm and onto the vertical wall with limited cooling capability. On the other hand, if the ion extractor is moved further away from the EC entrance diaphragm, its reflecting field in the region of the electron beam divergence can become acceptable and the elimination of the electron repeller can be done with the existing electron collector. A proposed modification of the EC electrode system is presented in Fig. 5.

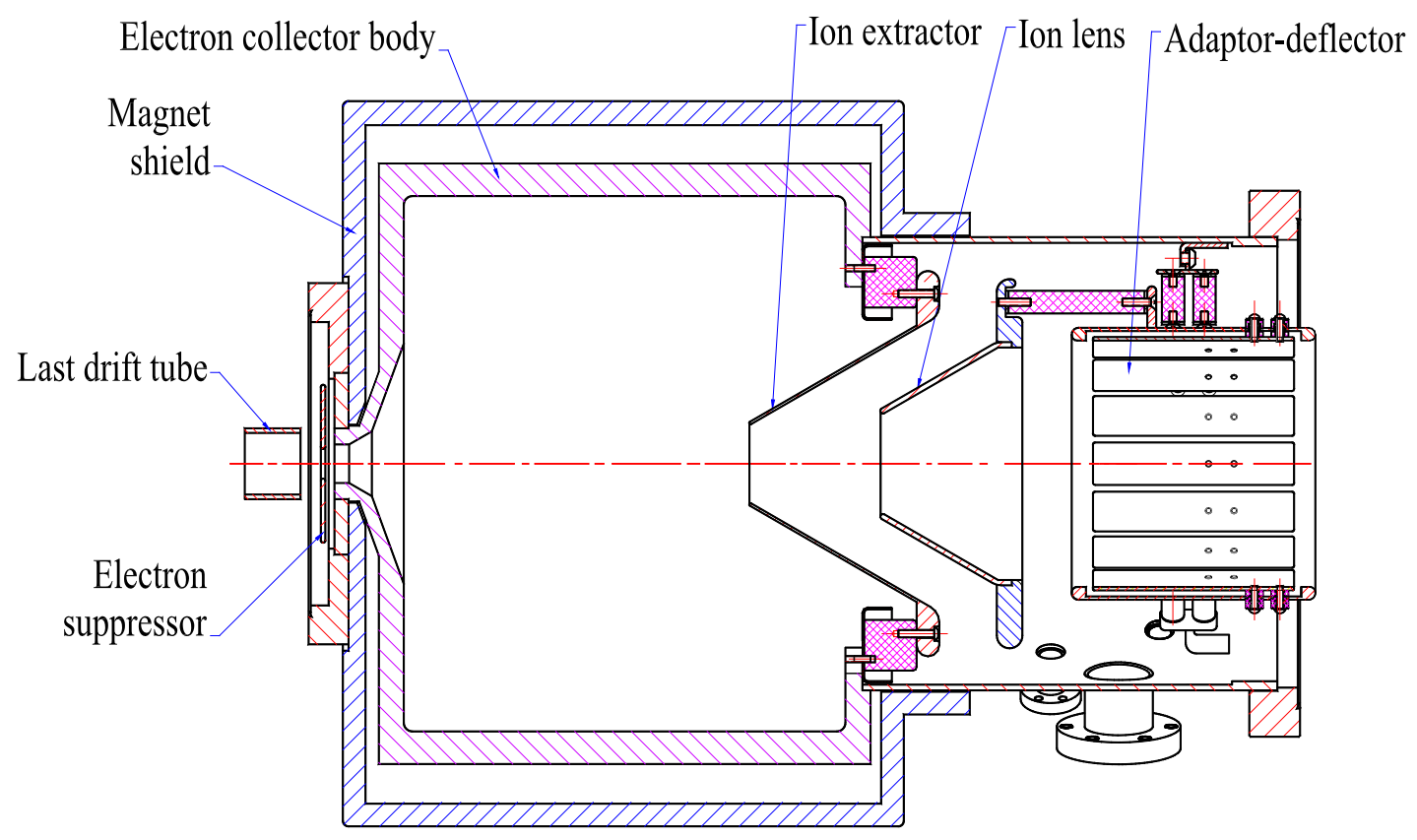

Fig. 5. Electrode geometry of the modified RHIC EBIS electron collector. 
Considering that the properties of the existing ion extraction/ion injection EC optics are acceptable, the general structure of the electrodes remains the same with some modification in dimensions and positions. It was possible to preserve the existing 16-pole adaptor/deflector.

The new ion extractor has approximately the same diameter of the entrance aperture (44 $\mathrm{mm})$ and the aperture of the new ion lens is larger taking into account an increased initial ion beam diameter compare to the existing optics. The HV holdoff of electrodes in an upgraded version is expected to be better because of an improved supports and reduced critical electric fields.

The simulated trajectories of the primary and backscattered electrons in a modified EC are presented in Fig. 6.

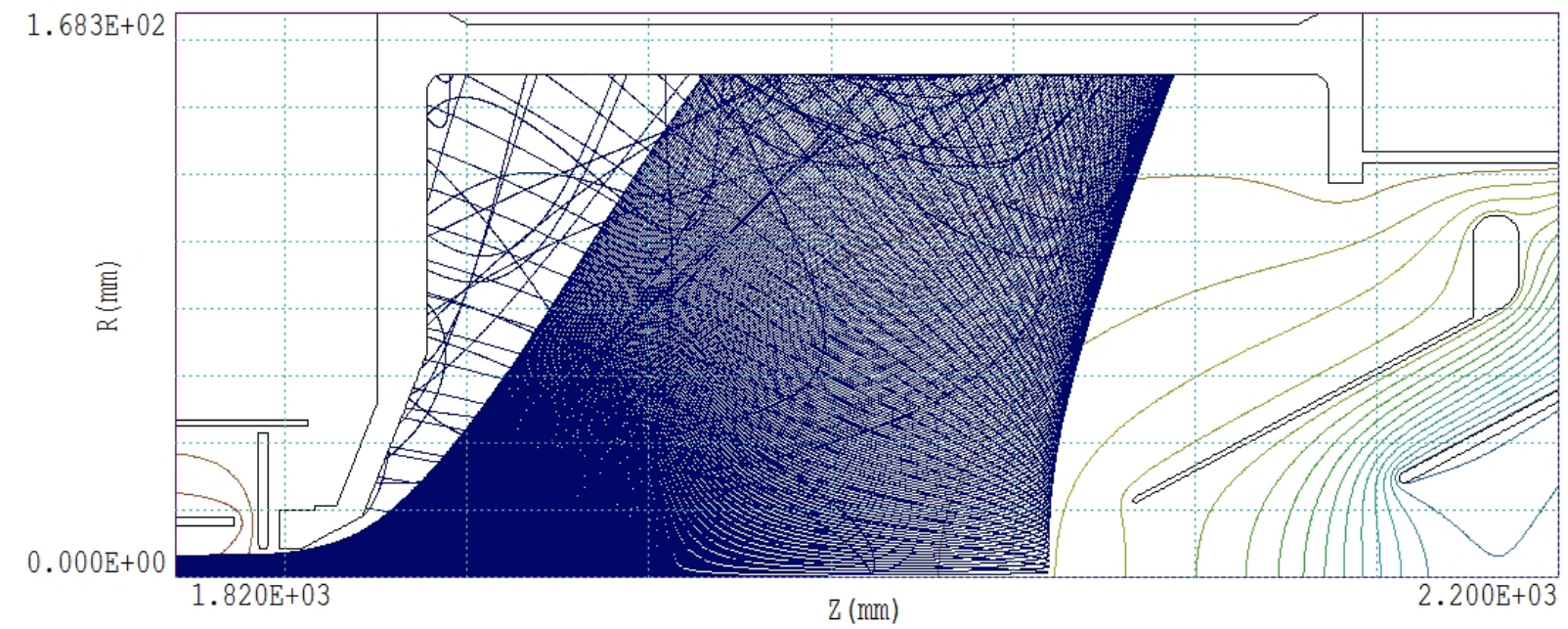

Fig. 6. Simulated trajectories of the primary electrons and of the first generation of backscattered electrons in RHIC EBIS EC with new optical geometry. $\mathrm{I}_{\mathrm{el}}=10.0 \mathrm{~A}, \mathrm{E}_{\mathrm{el}}=11.0 \mathrm{keV}$.

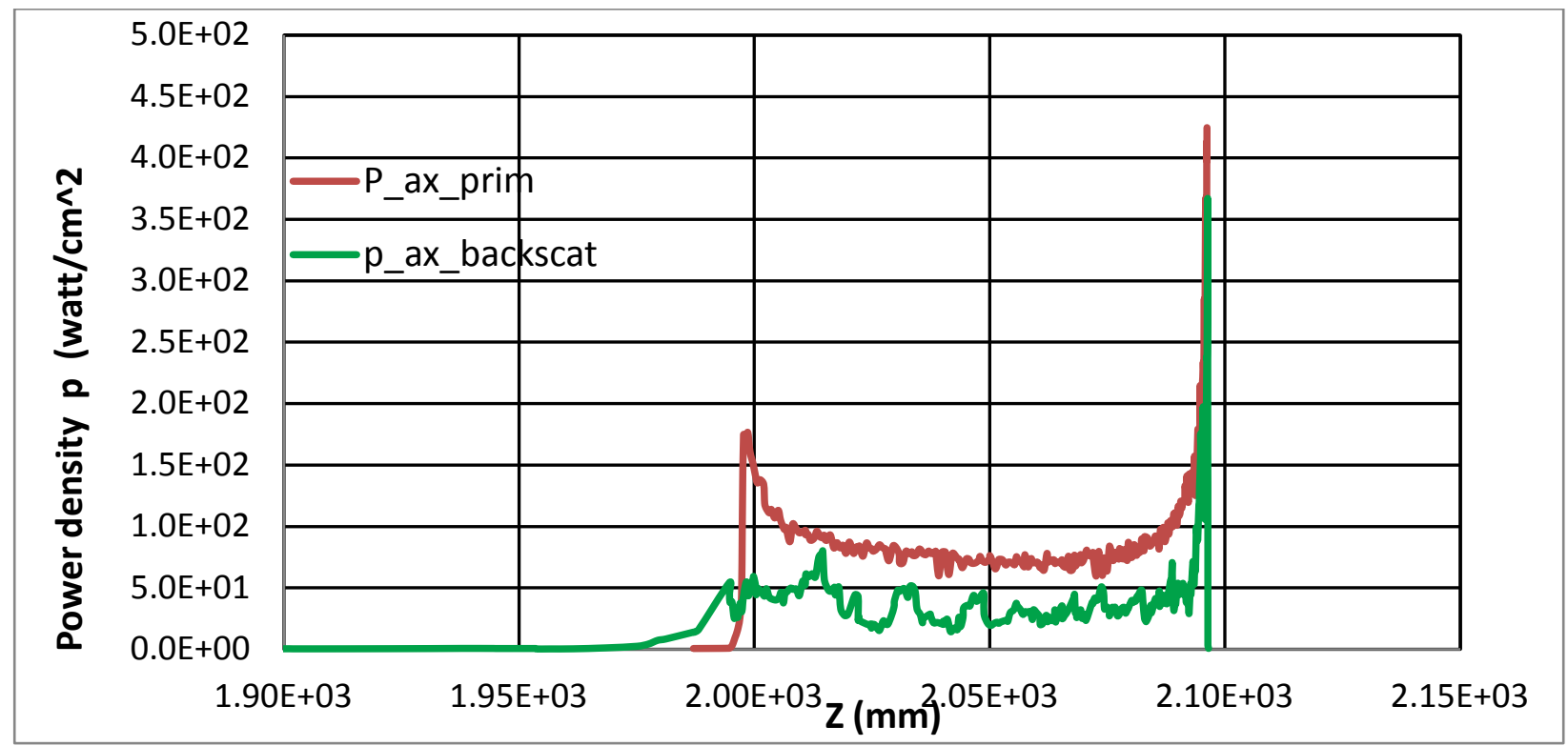

Fig. 7. Axial power density distributions of the primary and backscattered electrons in a new geometry of EC optics. Primary beam parameters: $\mathrm{I}_{\mathrm{el}}=10.0 \mathrm{~A}, \mathrm{E}_{\mathrm{el}}=11.0 \mathrm{keV}$. 
Similar to a previous case, the deposition of the backscattered electrons is mostly limited to the areas, which are bombarded by the primary electrons. The power density distribution on the cylindrical surface for a new geometry is presented in Fig. 7. For electron current $10 \mathrm{~A}$ the power density distribution on the cylindrical surface of EC remains approximately the same for both versions of EC optics, even that it shifts axially forward in a new version compare to the existing one. No effect on the reflecting of the primary electron backwards, resulting from this modification has been observed.

\section{Conclusion}

The proposed modification of the RHIC EBIS electron collector with purpose to eliminate the hot electron repeller electrode can be done with the existing electron collector body and its vacuum chamber. The simulated power dissipation on the electron collector walls remains similar to the original EC and the simulated flux of the reflected electrons did not increase. The 16-pole adaptor deflector remains intact and only its position has been changed. The HV design is more robust than the original one. Since the structure of the ion optical element did not change, only the dimensions did, the effect of optical elements in a modified version of EC on the injected and extracted ion beams remains similar to the original one according to our initial plans.

\section{References}

1. Alexander Pikin, Ahovi Kponou, Louis Snydstrup, Optical, Thermal and Stress Simulations of a 300-kwatt Electron Collector, BNL Tech Note C-A/AP/\#246, 2006

2. Alexander Pikin, James G. Alessi, Edward N. Beebe, Ahovi Kponou, Krsto Prelec, John Ritter, Louis Snydstrup, Analysis of a possible 20 A electron gun and collector design for the RHIC EBIS, Journal of Physics: Conference series 2, pp. 28-34, 2004

3. J. G. Alessi, E. Beebe, D. Graham, A. Kponou, K. Prelec, J. Ritter, V. Zajic, Design of an EBIS for RHIC, PAC-2003 Proceedings, V. 1, pp. 89-91, 2003

4. A. Pikin, J. G. Alessi, E. N. Beebe, A. Kponou, R. Lambiase, R. Lockey, D. Raparia, J. Ritter, L. Snydstrup and Y. Tan, RHIC EBIS: basics of design and status of commissioning, Proceedings of the 11-th International Symposium on Electron Beam ion Sources and Traps, Stockholm, 2010, in JINST, 5, C09003, 2010

5. A. Pikin, j. Alessi, E. Beebe, A. Kponou, M. Okamura, Ion optics of RHIC Electron Beam Ion Source, Rev. Sci. Instrum, 83, 02A50, 2012 